\title{
A Class of Positive Stable Matrices*
}

\section{David Carlson**}

(September 11, 1973)

\begin{abstract}
A square complex matrix is positive sign-symmetric if all its prıncıpal minors are positive, and all products of symmetrically-placed minors are nonnegative. It is proved that every positive sign-symmetric matrix is positive stable.
\end{abstract}

Key words: Positive stable matrix; sign-symmetry; spectrum.

1. Notation. For fixed $n$, let

$$
Q_{n}=\left\{\left(i_{1}, i_{2}, \ldots, i_{k}\right) \mid 1 \leqslant i_{1}<i_{2}<\ldots .<i_{k} \leqslant n\right\} .
$$

If $\alpha=\left(i_{1}, i_{2}, \ldots, i_{k}\right) \epsilon Q_{n}$, then $|\alpha|=k$. Given $A \epsilon C^{n, n}$ and $\alpha, \beta \epsilon Q_{n}$, by $A(\alpha, \beta)$ we mean the minor of $A$ whose rows are indexed by $\alpha$ and whose columns are indexed by $\beta$. We can now formally define $A \epsilon C^{n, n}$ to be positive sign-symmetric if

(1) $A(\alpha, \alpha)>0$ for all $\alpha \epsilon Q_{n}$,

(2) $A(\alpha, \beta) A(\beta, \alpha) \geqslant 0$ for all $\alpha, \beta \epsilon Q_{n},|\alpha|=|\beta|$.

It is obvious that hermitian positive definite matrices and totally positive matrices are positive signsymmetric. Also, it is well known that they have all positive characteristic roots (see also [9] ${ }^{1}$ ). This last is not true for all positive sign-symmetric matrices; as an example, take

$$
A=\left(\begin{array}{lll}
4 & 1 & 1 \\
2 & 4 & 2 \\
2 & 1 & 4
\end{array}\right)
$$

The characteristic roots of this matrix are, approximately, 6.85 and $2.58 \pm 0.28 i$.

It is perhaps of interest, however, that all positive sign-symmetric matrices are positive stable, i.e., all their characteristic roots have positive real parts.

THEOREM. Every complex matrix which is positive sign-symmetric is positive stable.

Proof. Given $B \epsilon C^{n, n}$ which is positive sign-symmetric. Let $S=B^{2}$; by the Cauchy-Binet formula and (1) and (2),

$$
S(\alpha, \alpha)=\Sigma_{|\beta|=|\alpha|} B(\alpha, \beta) B(\beta, \alpha)>0 \text { for all } \alpha \epsilon Q_{n}
$$

By a result of Fiedler and Pták [5], since all principal minors of $S$ are positive, no real characteristic root of $S$ is nonpositive. Since the characteristic roots of $S$ are the squares of the characteristic roots of $B, B$ can have no characteristic roots on the imaginary axis.

AMS Subject Classification: 15A18, 15A57, 65F15.

*An invited paper.

***Present address: Mathematics Department Oregon State University, Corvallis, Oregon 97331

${ }^{1}$ Figures in brackets indicate the literature references at the end of this paper. 
Now let $A \epsilon C^{n, n}$ be a positive sign-symmetric matrix. Since all its principal minors are positive, by a result of Fisher and Fuller [6] and Ballantine [1], there exists a $D=\operatorname{diag}\left(d_{1}, d_{2}, \ldots, d_{n}\right) \epsilon C^{n, n}$, $d_{i}>0, i=1, \ldots, n$, for which $A D$ is positive stable. Let $D_{t}=(1-t) I+t D, 0 \leqslant t \leqslant 1$; all $D_{t}$ are diagonal matrices, with positive diagonal entries, and $D_{0}=I, D_{1}=D$. It follows that each matrix $A D_{t}$ is positive sign-symmetric, and hence can have no characteristic roots on the imaginary axis. Since $A D$ is positive stable, and the characteristic roots of $A D_{t}$ are continuous functions of $t, A$ must also be positive stable.

We define $A \epsilon C^{n, n}$ to be positive weakly sign-symmetric if (1) and

(3) $A(\alpha, \beta) A(\beta, \alpha) \geqslant 0$ for all $\alpha, \beta \epsilon Q_{n},|\alpha|=|\beta|=|\alpha \cap \beta|+1$,

i.e., whenever all but one of the indices in $\alpha$ and $\beta$ are common to both. A number of interesting inequalities are known to hold for the principal minors of such matrices; cf. [3], [4], [8]. It is known that nonsingular $M$-matrices, as well as hermitian positive definite and totally positive matrices, are positive weakly sign-symmetric (cf. [2]); and all these matrices are positive stable. We conjecture that all positive weakly sign-symmetric matrices are also positive stable. The Routh-Hurwitz conditions (cf. [7], p. 194-5) can be used to prove that the conjecture is true for $n \leqslant 4$ (the case $n=3$ is also proved in [2]). An analogous proof for the general case appears hopelessly complicated.

\section{References}

[1] Ballantine, C. S., Stabilization by a diagonal matrix, Proc. Amer. Math. Soc. 25, 728-734 (1970).

[2] Carlson, David, Weakly sign-symmetric matrices and some determinantal inequalities, Colloq. Math. 17, 123-129 (1967).

[3] Fan, Ky, Subadditive functions on a distributive lattice and an extension of Szász's inequality, J. Math. Anal. Appl. 18, 262-268 (1967).

[4] Fan, Ky, An inequality for subadditive functions on a distributive lattice, with application to determinantal inequalities, Lin. Alg. Appl. 1,33-38 (1968).

[5] Fiedler, M., and Pták, V., On matrices with non-positive off-diagonal elements and positive principal minors, Czech, Math. Journal 12,382-400 (1962).

[6] Fisher, M. E., and Fuller, A. T., On the stabilization of matrices and the convergence of linear iterative processes, Proc. Camb. Philos. Soc. 54, 417-425 (1958).

[7] Gantmacher, F. R., The Theory of Matrices, Chelsea, New York, 1959, Vol. II, 27o p.

[8] Koteljanskii, D. M., A property of sign-symmetric matrices, Uspehi Mat. Nauk (N.S.) 8, 163-7 (1953).

19] Koteljanskiî, D. M., Some sufficient conditions for reality and simplicity of the spectrum of a matrix, Mat. Sb. N.S. 36, 163-168(1955).

(Paper 78B1-391) 